Lu-San Chen

Nagoya Math. J.

Vol. 37 (1970), 1-4

\title{
NOTE ON THE BEHAVIOR OF SOLUTIONS OF PARABOLIC EQUATIONS WITH UNBOUNDED COEFFIGIENTS*)
}

\author{
LU-SAN CHEN
}

\section{To Professor Katuzi Ono on the occasion of his 60th birthday}

1. Let

$$
L=\sum_{i, j=1}^{n} a_{i j} \frac{\partial^{2}}{\partial x_{i} \partial x_{j}}+\sum_{i=1}^{n} b_{i} \frac{\partial}{\partial x_{i}}+c-\frac{\partial}{\partial t}
$$

be a parabolic differential operator defined in $\Omega=R^{n} \times(0, \infty)$, where $R^{n}$ is the $n$-dimensional Euclidean space, the point $x \in R^{n}$ is represented by its coordinates $\left(x_{1}, \cdots, x_{n}\right)$ and $a_{i j}\left(=a_{j i}\right), b_{i}$ and $c$ are functions in $(x, t) \in \Omega$. We assume that there exist constants $k_{1}(>0), K_{1}, K_{2}(\geqslant 0), K_{3}(>0)$ and $K_{4}$ such that

$$
\left\{\begin{array}{l}
k_{1}\left(|x|^{2}+1\right)^{1-\lambda}|\xi|^{2} \leqslant \sum_{i, j=1}^{n} a_{i j} \xi_{i} \xi_{j} \leqslant K_{1}\left(|x|^{2}+1\right)^{1-\lambda}|\xi|^{2} \\
\left|b_{i}\right| \leqslant K_{2}\left(|x|^{2}+1\right)^{1 / 2},(1 \leqslant i \leqslant n) \\
c \leqslant-K_{3}\left(|x|^{2}+1\right)^{\lambda}+K_{4}
\end{array}\right.
$$

for some $\lambda \in(0,1]$.

Consider the Cauchy problem

$$
\left\{\begin{array}{l}
L u=0 \text { in } \Omega, \\
u(x, 0)=f(x) .
\end{array}\right.
$$

2. Throughout this note, we shall say that $u(x, t)$ is a solution of the problem (2) when $u(x, t)$ is continuous in $\bar{\Omega}=R^{n} \times[0, \infty)$, twice continuously differentiable in $\Omega$ and satisfies (2).

Received October 21, 1968

*) This research was supported by the National Science council in Taiwan. 
In this note we shall prove the following which is a general form of Krzyżanski's theorem [2].

Theorem. Let $u(x, t)$ be a solution of the Cauchy problem (2) and $|u(x, t)|$ $\leqslant K_{5} e^{\mu\left(|x|^{2}+1\right) \lambda}$ for some constants $K_{5}$ and $\mu$. Assume that the coefficients of $L$ satisfy (1). If the Cauchy data $f(x)$ is bounded in $R^{n}$ and if

$$
\frac{1}{2 K_{1}}\left[2 K_{1}(1-\lambda)-k_{1} n\right]\left(\sqrt{K_{2}^{2} n^{2}+4 K_{1} K_{3}}-K_{2} n\right)+K_{4}<0,
$$

then $u(x, t)$ tends to zero uniformly in $x \in R^{n}$ as $t$ tends to infinity.

In the case of the differential operator

$$
L_{0}=\sum_{i=1}^{n} \frac{\partial^{2}}{\partial x_{i}^{2}}+\left(-K_{3}|x|^{2}+K_{3}^{\prime}\right)-\frac{\partial}{\partial t},
$$

we may take $k_{1}=K_{1}=1, K_{2}=0, K_{4}=K_{3}^{\prime}-K_{3}$ and $\lambda=1$ in Theorem. So the solution $u(x, t)$ of the Cauchy problem

$$
\left\{\begin{array}{l}
L_{0} u=0 \text { in } \Omega, \\
u(x, 0)=f(x)
\end{array}\right.
$$

for a bounded Cauchy data $f(x)$ tends to zero uniformly in $x \in R^{n}$ as $t$ tends to infinity, if

$$
\sqrt{K_{3}} n>K_{3}^{\prime}-K_{3} \text {. }
$$

3. To prove our theorem, we use the following.

Lemma. Let $\alpha$ be a positive root of the quadratic equation $A X^{2}+B X+C=0$, where $B \geqslant 0$ and $C<0$. Then the function

$$
\varphi(t)=\alpha \tanh A \alpha t
$$

satisfies the inequality

$$
\varphi^{\prime}(t)+A \varphi^{2}(t)+B \varphi(t)+C \leqslant 0 .
$$

The proof is given by the direct calculation, so we may omit it here. Now we shall give the proof of Theorem.

Let $\varphi(t)$ and $\psi(t)$ be functions twice continuously differentiable in $[0, \infty)$. Putting

$$
H(x, t)=\exp \left[-\varphi(t)\left(|x|^{2}+1\right)^{2}+\psi(t)\right]
$$


we see from (1) that

$$
\begin{aligned}
\frac{L H}{H}= & 4 \lambda^{2} \varphi^{2}(t)\left(|x|^{2}+1\right)^{2 \lambda-2} \sum_{i, j=1}^{n} a_{i j} x_{i} x_{j}+4 \lambda(1-\lambda) \varphi(t)\left(|x|^{2}+1\right)^{\lambda-2} \sum_{i, j=1}^{n} a_{i j} x_{i} x_{j} \\
& -2 \lambda \varphi(t)\left(|x|^{2}+1\right)^{\lambda-1} \sum_{i=1}^{n}\left(a_{i i}+b_{i} x_{i}\right)+c+\varphi^{\prime}(t)\left(|x|^{2}+1\right)^{\lambda}-\psi^{\prime}(t) \\
\leqslant & \left(|x|^{2}+1\right)^{\lambda}\left[\varphi^{\prime}(t)+4 K_{1} \lambda^{2} \varphi^{2}(t)+2 K_{2} n \lambda \varphi(t)-K_{3}\right] \\
& +\left[4 \lambda(1-\lambda) K_{1} \varphi(t)-2 \lambda k_{1} n \varphi(t)+K_{4}-\psi^{\prime}(t)\right] .
\end{aligned}
$$

So, if

$$
\varphi(t)=\alpha \tanh 4 K_{1} \lambda^{2} \alpha t
$$

for the positive root

$$
\alpha=\frac{-K_{2} n+\sqrt{K_{2}^{2} n^{2}+4 K_{1} K_{3}}}{4 K_{1} \lambda}
$$

of the quadratic equation $4 K_{1} \lambda^{2} X^{2}+2 K_{2} n \lambda X-K_{3}=0$, then we see from Lemma that

$$
\varphi^{\prime}(t)+4 K_{1} \lambda^{2} \varphi^{2}(t)+2 K_{2} n \lambda \varphi(t)-K_{3} \leqslant 0 .
$$

Further, it is easy to see that

$$
\psi(t)=\frac{2 K_{1}(1-\lambda)-k_{1} n}{2 K_{1} \lambda} \log \left[\cosh 4 K_{1} \lambda^{2} \alpha t\right]+K_{4} t
$$

satisfies

$$
4 \lambda(1-\lambda) K_{1} \varphi(t)-2 \lambda k_{1} n \varphi(t)+K_{4}-\psi^{\prime}(t)=0
$$

for $\varphi(t)$ given by (5). Thus $H(x, t)$ given by (4) for $\varphi(t)$ in (5) and $\psi(t)$ in (6) satisfies

$$
L H \leqslant 0
$$

in $\Omega$. It is evident that $H(x, 0)=1$.

As the Cauchy data $f(x)$ in (2) is bounded, we may assume $|f(x)|<M$ in $R^{n}$. If we put

$$
w_{+}(x, t)=M H(x, t)+u(x, t)
$$

then $L w_{+}=M L H+L u=M L H \leqslant 0$ in $\Omega$ and $w_{+}(x, 0)=M+f(x) \geqslant 0$. Moreover, we have clearly $\left|w_{+}(x, t)\right| \leqslant K_{4}^{\prime} e^{\mu \prime}\left(|x|^{2}+1\right)^{\lambda}$ in $\Omega$ for some constants $K_{4}^{\prime}$ 
and $\mu^{\prime}$. The maximum principle due to Bodanko [1] implies that $w_{+}(x, t) \geqslant 0$ in $\Omega$, that is,

$$
-M H(x, t) \leqslant u(x, t)
$$

in $\Omega$. We apply the same argument to $w_{-}(x, t)=M H(x, t)-u(x, t)$ as the above, we get

$$
M H(x, t) \geqslant u(x, t)
$$

Thus we obtain

$$
\begin{aligned}
|u(x, t)| & \leqslant M H(x, t) \\
& \leqslant M \exp \left\{\left[4 K_{1} \lambda(1-\lambda) \alpha-2 \lambda k_{1} n a+K_{4}\right] t\right\}
\end{aligned}
$$

for $\alpha$ in (5) throughout $\Omega$. From the assumption (3), it is obvious that $u(x, t)$ tends to zero uniformly in $x \in R^{n}$ as $t$ tends to infinity.

Remark. Krzyżański [2] considered the case $\lambda=1$ in our Theorem and gave an analogous result.

\section{RefERENCES}

[ 1 ] W. Bodanko, Sur le problème de Cauchy et les problèmes de Fourier pour les equations paraboliques dans un domaine non borné, Ann. Polo. Math., 18 (1966), 79-94.

[2] M. Krzyżański, Evaluations des solutions de l'equation lineaire de type paraboliques à coefficients non borné, Ann. Polo. Math., 11 (1961-62), 253-260.

Department of Mathematics

Taiwan Provincial Cheng-Kung University, China,

and

Mathematical Institute

Tohoku University, Sendai, Japan 